\title{
Panorama de la robótica educativa a favor del aprendizaje STEAM
}

\author{
María Obdulia González Fernández iD \\ Dpto. de Ingeniarias, Centro Universitario de los Altos, Universidad de Guadalajara. Tepatitlán. México. \\ ogonzalez@cualtos.udg.mx
}

\author{
Yadira Alejandra Flores González (D), Claudia Muñoz López iD \\ Ingeniería en Computación, Centro Universitario de los Altos, Universidad de Guadalajara. Tepatitlán. \\ México.yadira.glores@alumnos.udg.mx, claudia.munoz2473@alumnos.udg.mx
}

[Recibido: 7 abril 2020. Revisado: 27 mayo 2020. Aceptado: 14 septiembre 2020]

\begin{abstract}
Resumen: Este artículo presenta un panorama de las diversas investigaciones e intervenciones educativas referidas a la robótica educativa y aprendizaje STEAM. Para esto se llevó a cabo una investigación documental, cualitativa interpretativa, en bases de datos especializadas. Se utilizaron los descriptores: robótica educativa, aprendizaje-robótica, STEAM-robótica, habilidades STEAM y pensamiento computacional. Como resultado se obtuvo el análisis de 105 documentos publicados del 2005 al 2019, se clasificaron de acuerdo con el idioma, ubicación geográfica, nivel educativo, tipo de estudio, temporalidad y tecnología utilizada. Las experiencias educativas se registran principalmente en el nivel primaria y secundaria. Se destacan los beneficios en cuanto al desarrollo de competencias de comunicación, trabajo en equipo, creatividad y resolución de problemas. Las metodologías didácticas empleadas son aprendizaje basado en problemas, proyectos y aprendizaje colaborativo, vivencial y lúdico, relacionadas con teorías construccionistas.
\end{abstract}

Palabras clave: robótica educativa; STEAM; aprendizaje; construccionismo; pensamiento computacional

\section{Overview of Educational Robotics for STEAM Learning}

Abstract: This article presents an overview of the various educational research and learning interventions referred to educational robotics and STEAM learning. For this, qualitative interpretive documentary research was carried out in specialized databases. Descriptors: educational robotics, learning-robotics, STEAM-robotics, STEAM skills, and computational thinking. As a result, the analysis of 105 documents published from 2005 to 2019 was obtained, they were organized according to language, geographic location, educational level, temporality, kind of investigation, and technology used. The educational experiences are mainly recorded in elementary and middle school levels. The benefits in terms of developing communication skills, teamwork, creativity, and problem-solving are highlighted. The didactic methodologies employed are problem-based learning, projects, and collaborative learning, experiential and playful learning, associated with constructionist theories.

Keywords: educational robotics, STEAM, learning, constructionism, computational thinking.

Para citar este artículo: González Fernández M. O., Flores González Y. A. y Muñoz López C. (2021) Panorama de la robótica educativa a favor del aprendizaje STEAM. Revista Eureka sobre Enseñanza y Divulgación de las Ciencias 18(2), 2301. doi: 10.25267/Rev_Eureka_ensen_divulg_cienc.2021.v18.i2.2301

\section{Introducción}

La interacción humana con la tecnología en la actualidad es una tendencia casi natural, ya que se encuentra presente en la mayoría de las actividades del ser humano. En el caso del sistema educativo ocurre un fenómeno en crecimiento, que es el uso de la robótica educativa para el desarrollo de habilidades en los niños y jóvenes en pro del aprendizaje. Se han detectado varios enfoques, entre ellos usar el robot como una herramienta para que el educando aprenda a construirlo, programarlo y así genere nuevos prototipos. Todo esto en pro del desarrollo de habilidades del pensamiento lógico-matemático, el algorítmico y habilidades como la creatividad, el trabajo colaborativo, la comunicación, entre otras.

\author{
Revista Eureka sobre Enseñanza y Divulgación de las Ciencias \\ Universidad de Cádi\%. APAC-Eureka. ISSN: 1697-011X \\ bttp://doi.org/10.25267/Rev_Eureka_ensen_divulg_cienc.2021.v18.i2.2301 \\ http:/ / reuredc.uca.es
}


La robótica educativa tiene sus orígenes alrededor de los años 60's, a partir de las investigaciones del Instituto Tecnológico de Massachusetts (MIT) que construyó los primeros robots para ser manipulados y programados por los niños. Esto se ha popularizado en la última década, para el aprendizaje activo y la enseñanza interdisciplinaria como matemáticas, ciencia, tecnología y últimamente el desarrollo de la creatividad y arte (STEAM). Cabe mencionar que el acrónimo de STEAM fue utilizado por primera vez por Yakman (2008) quien bajo el principio de interdisciplinariedad, introdujo la inicial de ARST dentro del acrónimo de STEM.

De acuerdo con Romero et al. (2014) la integración de la robótica educativa en los currículos escolares de educación básica, incentiva el conocimiento tecnológico, para elevar la calidad de la educación. Se destacan los esfuerzos por integrar la robótica educativa en países como Alemania, Inglaterra, Italia, España, Canadá y Estados Unidos de América. Mientras que México ha iniciado en adoptar dicha tecnología en la educación primaria y secundaria. Por citar algunos, se encuentra que la Secretaría de Educación Pública en algunos estados de la república mexicana ha realizado pruebas pilotos en escuelas públicas con el objetivo de generar estudios para medir el impacto del uso del robot con fines didácticos y valorar la pertinencia en pro de su integración en el currículum para consolidar las habilidades de ciencia, matemáticas y tecnología. Además de acciones como cursos extracurriculares y ferias de robótica que ofrecen instituciones externas a la educación formal.

Si bien existen varios estudios que han presentado estados de arte de la robótica educativa como el caso de (Herrara y Rincón 2012, Romero et al. 2014). En el ámbito de pensamiento computacional González (2018) presenta un estudio del estado del arte del aprendizaje en edades tempranas. Esta autora argumenta que existen muy pocos estudios relacionados con la incorporación efectiva en el currículo escolar. Más, sin embargo, la intención de este trabajo es presentar un panorama integrado de las diversas investigaciones en torno a tres temáticas que están íntimamente relacionadas como son la robótica educativa, el aprendizaje STEAM y el pensamiento computacional, ya que la mayoría de los autores han estudiado dichos tópicos por separado y realizando una combinación siempre al entorno de la robótica.

Si bien el trabajo de Suárez et al. (2018) presentan una integración del pensamiento computacional con la robótica y da una pincelada del aprendizaje STEM, hace falta profundizar en los diferentes estudios que se han realizado en estos tres ámbitos.

Para esto es importante reconocer que el robot como herramienta para generar mediaciones a través de estrategias de enseñanza constructivas. Este tipo de acciones requiere de tiempo y formación docente; material didáctico para las lecciones, acorde y accesible tanto para educadores como para alumnos (Mataric et al. 2007).

La adecuada integración de la robótica educativa es beneficiosa para el aprendizaje, como lo señalan Bravo y Forero (2012) el uso del robot como instrumento didáctico desarrolla habilidades y competencias al dar respuestas a problemas multidisciplinarios. También desarrolla destrezas tecnológicas para afrontar los retos actuales. Otra de las ventajas es que genera expectativas y motivación del estudiante al facilitar la adquisición de conocimiento. En este mismo sentido Márquez y Ruiz (2014) señalan que el uso de los robots en la educación de los niños promueve la creatividad y la motivación, que, a posterior, le permitirá desarrollar habilidades cognitivas y manuales.

\section{Metodología}

Para realizar este trabajo se optó por una investigación documental de tipo cualitativo-interpretativo para generar un panorama actualizado del estado que guarda la robótica educativa y su relación con el aprendizaje STEAM. Todo esto mediante un estudio retrospectivo a partir de la valoración y caracterización de diversas investigaciones (Galeano-Marín y Vélez 2002). Esta recopilación de experiencias, permitió identificar, evaluar y determinar las tendencias de integración de dicha herramienta en pro del aprendizaje. 


\section{Instrumentos}

Se utilizaron la matriz bibliográfica y de contenido propuestas por Gómez et al. (2015), la primera logró ordenar los documentos de acuerdo con los criterios de autor, título del trabajo, palabras claves y resumen del documento. Mientras que la matriz de contenido realizada en Excel clasificó al autor, país, tecnología, metodología didáctica, nivel educativo, habilidades y tipo de documento.

\section{Procedimiento}

Para la construcción de este estado del arte se aplicó el procedimiento propuesto por Londoño et al. (2016) quienes sugieren que el análisis documental parte de dos procesos como son heurística y hermenéutica con la finalidad de explicar e interpretar las relaciones que existen en los diferentes documentos. Ver figura 1.

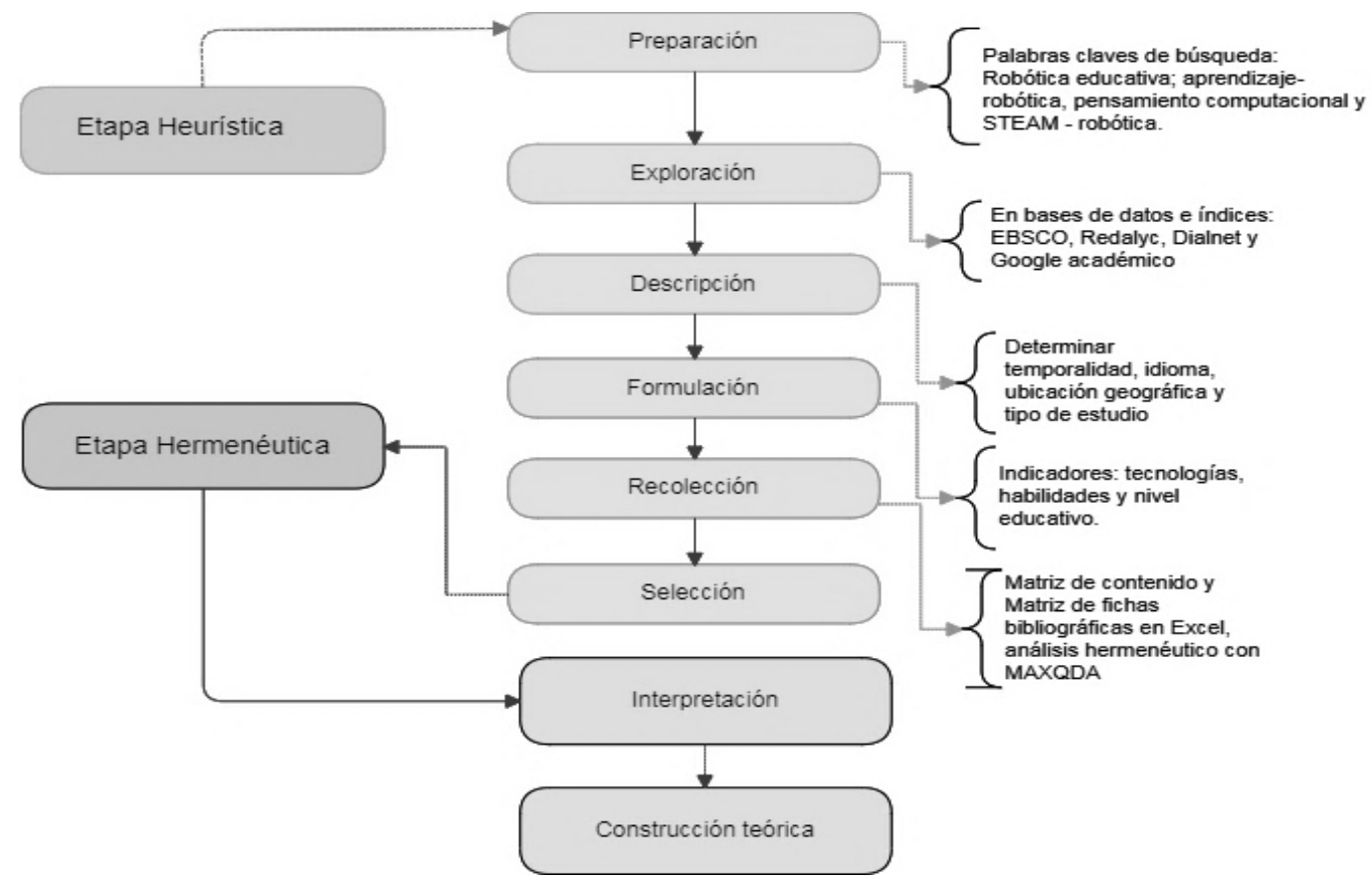

Figura 1. Ruta metodológica adaptada de Londoño, Maldonado y Calderón (2016).

La etapa heurística permitió la búsqueda y compilación de información a partir de diversas fuentes como bases de datos especializadas e índices como: EBSCO, Redalyc, Dialnet y Google académico, de diversas experiencias de investigaciones orientadas a la integración de la robótica educativa en el aula. Se utilizó las palabras claves de robótica educativa, aprendizaje-robótica, STEAM-robótica, habilidades STEAM y pensamiento computacional.

Para la selección y acopio de los documentos se consideró los criterios de inclusión como: Prestigio de la revista, el periodo de tiempo, nivel educativo, tipo de publicación e idioma inglés o español. Cabe aclarar que se seleccionaron seis documentos en idioma portugués por su trascendencia en otros estudios. Además de contar con la pauta de descarte de los documentos que contarán con las peculiaridades de:

- Documentos de carácter técnico de un robot

- Documentos tipo blog

- Los resúmenes de artículos

- Artículos de libros

- Libros que no fueran resultado de una investigación

- Artículo del área de ingenierías

- Artículos del nivel de pregrado y postgrado 
Las fuentes recuperadas se organizaron en una matriz de contenido y fichas bibliográficas. En esta etapa se utilizó el gestor de referencias Mendeley y el software de análisis cualitativo MAXQDA. Este último cuenta con una herramienta para el manejo de datos bibliográficos y un conjunto de herramientas visuales para representar los resultados. Esto permitió generar las redes semánticas a partir de los contenidos de los documentos analizados. De acuerdo a Rädiker y Kuckartz (2019) MAXQDA es una herramienta que proporciona un valioso apoyo en la revisión de la literatura, al generar un sistema de códigos y variables documentales a partir de los metadatos de los archivos.

\section{Resultados}

Se recolectaron y analizaron un total de 105 documentos publicados del 2005 al 2019. De los cuales 51 documentos son referentes a robótica educativa, Educational Robotics, 36 a robótica y habilidades STEAMSTEM y 18 se refieren al pensamiento computacional. De los cuales 74 son en idioma español, 25 en inglés y 6 en portugués.

En la figura 2 se puede observar el modelo de códigos procesado en MAXQDA, donde la dimensión de la línea muestra las frecuencias de las coincidencias en las palabras claves. Además de que se muestran los principales autores de los trabajos asociados a dicho término.

El campo de estudio de la robótica educativa se caracteriza por ensayos y experiencias en distintas instituciones y niveles educativos. De acuerdo con Girvan et al. (2017) este es un campo dividido entre investigaciones de desarrollo informático e ingeniería que plantean distintos desarrollos de robots educativos.

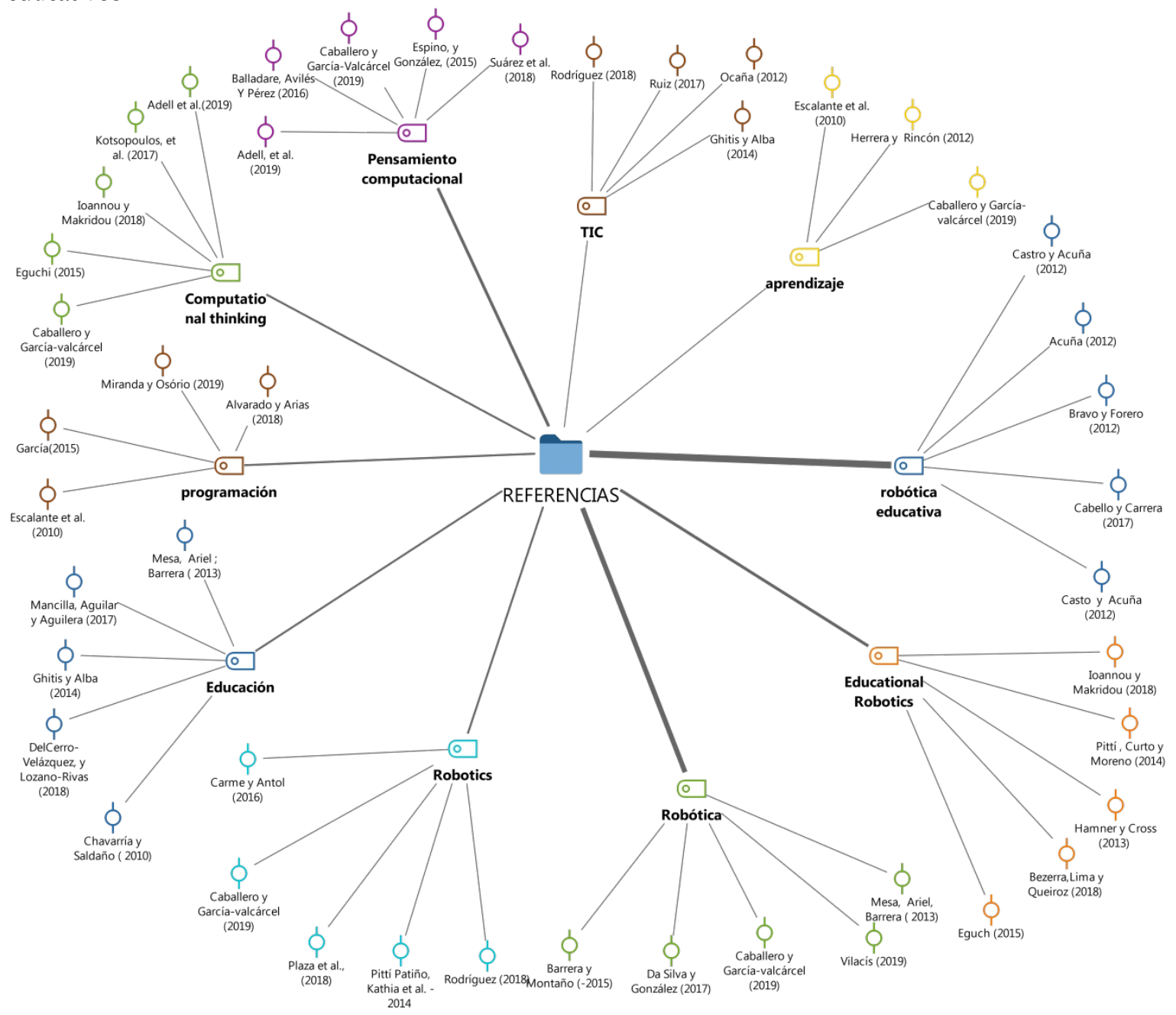

Figura 2. Modelo de códigos de las palabras claves a partir del sistema de referencias (Elaboración propia) 
Por otra parte, una línea de investigación más amplia describe el impacto de las actividades de robótica educativa relacionada con las habilidades de STEAM en el niño, que a menudo carecen de fundamento pedagógico. Por eso la necesidad de establecer un puente entre la parte tecnológica y la pedagógica.

Al clasificar los documentos en cuanto al tipo de estudio, se encontró que el $42 \%$ refieren a informes de investigaciones, el 33\% son artículos de revisión documental, el 13\% corresponden a experiencias docentes y el $12 \%$ no especifican el tipo de investigación.

En la figura 3 se muestra que el $44 \%$ son artículos de estudios de casos con niños en rangos de edades entre seis y 12 años de edad, en nivel primaria y $24 \%$ son de secundaria.

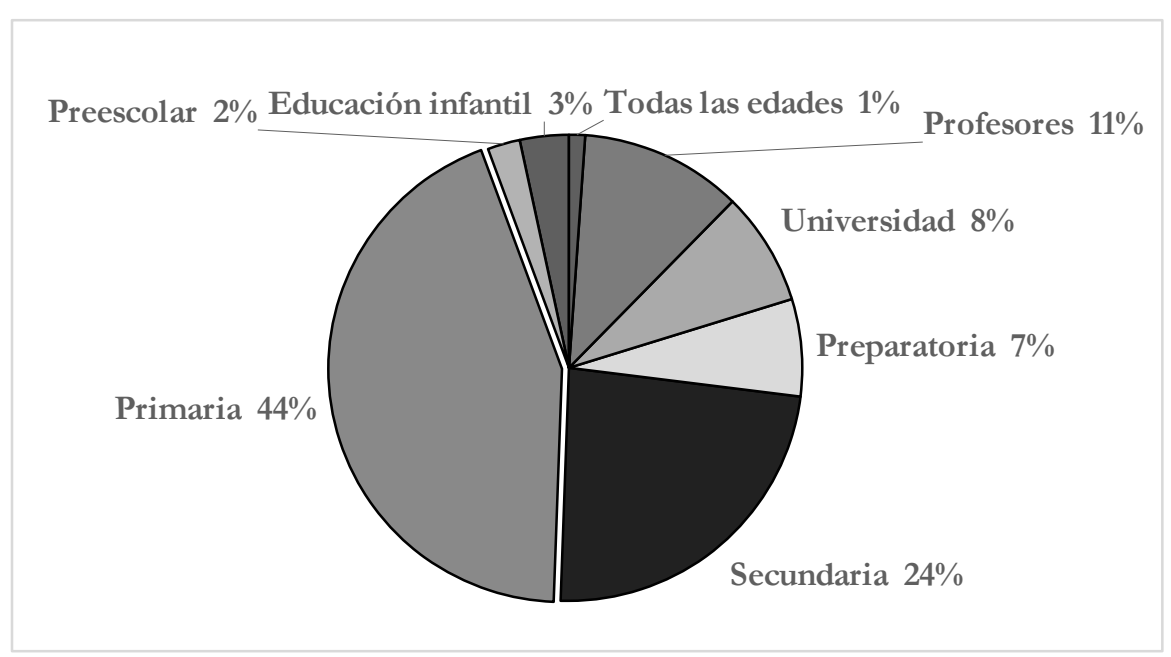

Figura 3. Distribución de investigaciones por nivel educativo (Elaboración propia)

La principal tecnología que se utiliza es LEGO, en cuanto a lenguajes de programación se encuentra el software Scratch; cabe mencionar que éstas son muy variadas, dependiendo del país, el nivel y el propósito de la investigación. Se clasificaron con la etiqueta de "otros" aquellas tecnologías cuya presencia se encuentra en un sólo documento. Ver tabla 1.

Tabla 1. Distribución de tecnología por documento analizado

\begin{tabular}{|l|l|}
\hline \multicolumn{1}{|c|}{ Tecnología usada } & \multicolumn{1}{c|}{ Total } \\
\hline Lego & 21 \\
\hline Software Scratch & 13 \\
\hline Arduino & 5 \\
\hline Bee-Bot & 5 \\
\hline Software NXT-G & 2 \\
\hline Handy Cricket & 2 \\
\hline Gogo Board & 2 \\
\hline Otros & 40 \\
\hline No se específica & 15 \\
\hline Total & 105 \\
\hline
\end{tabular}

\section{Análisis del concepto de robótica educativa}

Primeramente, se presenta el análisis de los documentos cuyo propósito es ampliar el campo semántico y conceptual del término robótica educativa. En este sentido Ghitis y Alba (2014) la refieren con varias denominaciones como robótica pedagógica, robótica y educación, robótica 
aplicada a la educación. Es así que diversos autores coinciden que existen dos perspectivas para el término; el aprendizaje de la robótica como objeto de estudio y el aprendizaje con la robótica como una herramienta mediática para el aprendizaje (Candanedo 2012, López y Andrade 2013, Mubin et al. 2013, Pittí et al. 2014). Esta última perspectiva concibe un contexto de aprendizaje apoyado por las TIC, donde los estudiantes participan activamente en el desarrollo de actividades con robots a partir de las experiencias educativas lúdicas, constructivos a favor de un aprendizaje interdisciplinario. Por lo que el robot despierta el interés y motivación en los estudiantes.

El integrar la robótica educativa es una forma de generar la interdisciplinariedad, desde un enfoque de "ciencia, tecnología y sociedad" al interior del currículum escolar, sin prescindir de principios pedagógicos, objetivos, contenidos, metodología, recursos y la evaluación de aprendizajes (Chavarría y Saldaño 2010, Bravo y Forero 2012). Al ser una herramienta atractiva para niños y jóvenes produce ambientes en los que se activan los procesos cognitivos y sociales propios del aprendizaje significativo. Se caracteriza por confluir áreas como: matemáticas, física, electrónica, mecánica e informática. Viegas et al. (2017) explican que esto es debido a que en dicho entorno se puede conciliar lo concreto y lo abstracto en pro de la resolución de un problema.

Así mismo, se destacan los estudios que demuestran los beneficios de integrar la robótica educativa en áreas de ciencias y medio ambiente (Filipp et al. 2017, Aliaga et al. 2018); mientras que Ocaña (2012) en su estudio demostró que la robótica en el aula es una herramienta pedagógica que propicia la motivación, el desarrollo de competencias básicas y la adquisición de conocimientos tecnológicos y científicos en general.

En la figura 4 se puede observar el análisis de los principales términos asociados al concepto de robótica educativa. Esta se asocia a metodologías didácticas del aprendizaje basado en problemas, proyectos, el aprendizaje activo, colaborativo, vivencial y lúdico.

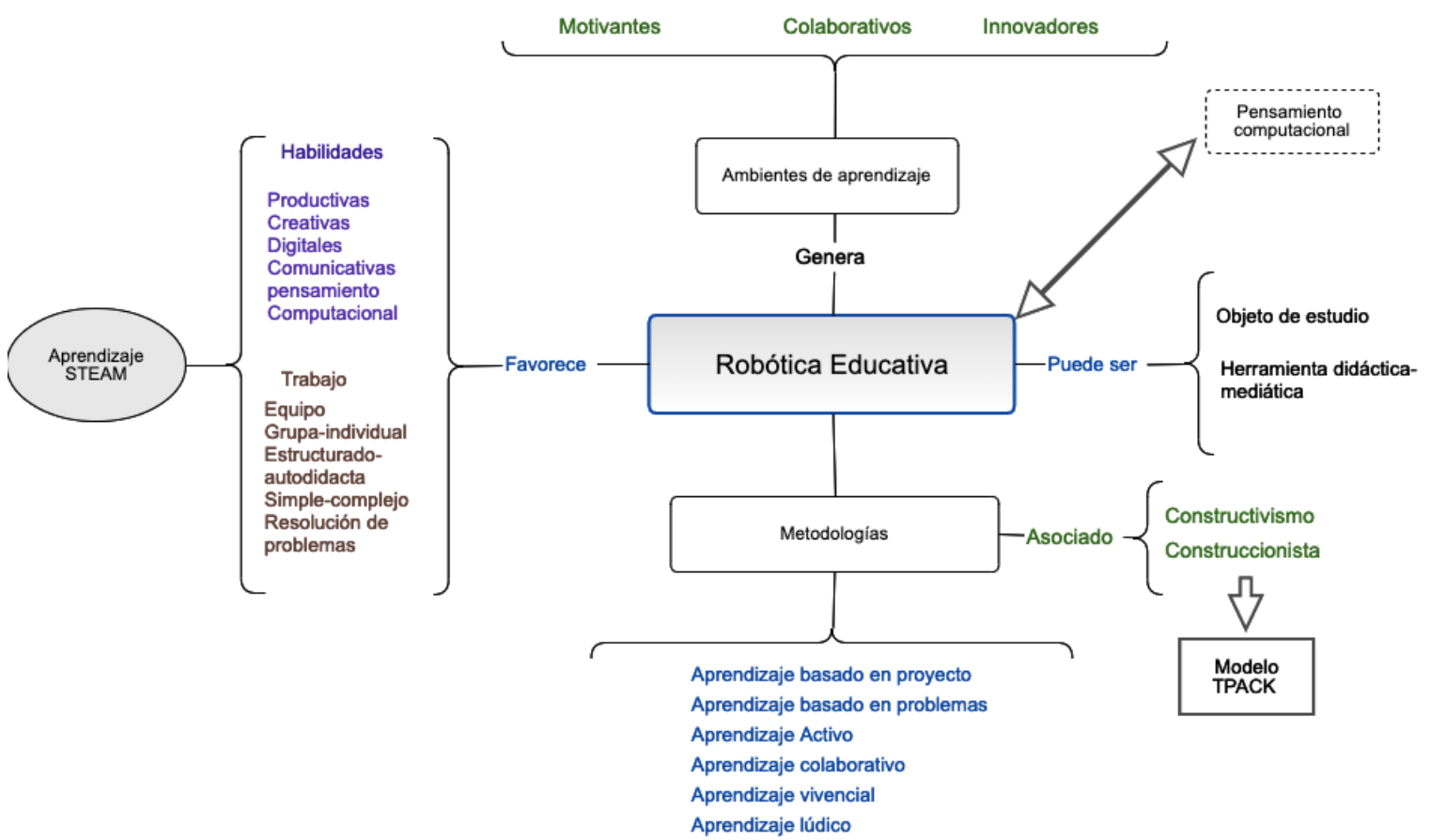

Figura 4. Mapa de términos asociados al concepto de robótica educativa (Elaboración propia)

Dichas metodologías se fundamentan en corrientes pedagógicas como el constructivismo y construccionismo (Mubin et al. 2013). Es así que el docente pone en marcha un conjunto de 
estrategias metodológicas que permite la integración del robot como una herramienta didáctica, en el que se generan espacios motivantes para la innovación y colaboración.

\section{Impacto de la robótica educativa en el aprendizaje}

Los diferentes estudios referencian que la robótica educativa integrada en contextos áulicos desarrolla habilidades en el estudiante de forma transversal e interdisciplinaria al currículo escolar. En un entorno formal educativo el robot educativo puede adquirir diversos roles, esto dependerá del contenido, la intención del docente y el tipo de estudiantes. En un principio puede adquirir un rol pasivo-instrumental, esto se da cuando los estudiantes crean o programan los robots. Por otro lado, puede tener un rol activo donde ahora el robot se convierte en un compañero y el robot puede ser mentor; como es el caso de la enseñanza de idiomas (Mubin et al. 2013).

En la figura 5 se muestra la clasificación de los diversos estudios e investigaciones en torno al desarrollo de habilidades a partir de la integración de la robótica.

La habilidad con mayor frecuencia es la creatividad, es así que Acuña (2012) retoma la robótica como un motor para la innovación, mediante el desarrollo de la creatividad y las actividades productivas. Por lo tanto, en el marco del aprendizaje del siglo XXI la robótica es una herramienta efectiva para mejorar las habilidades como la creatividad, la colaboración, el trabajo en equipo, la comunicación y las habilidades sociales. Por lo que la robótica es un recurso efectivo para preparar al estudiante (Khanlari 2016).

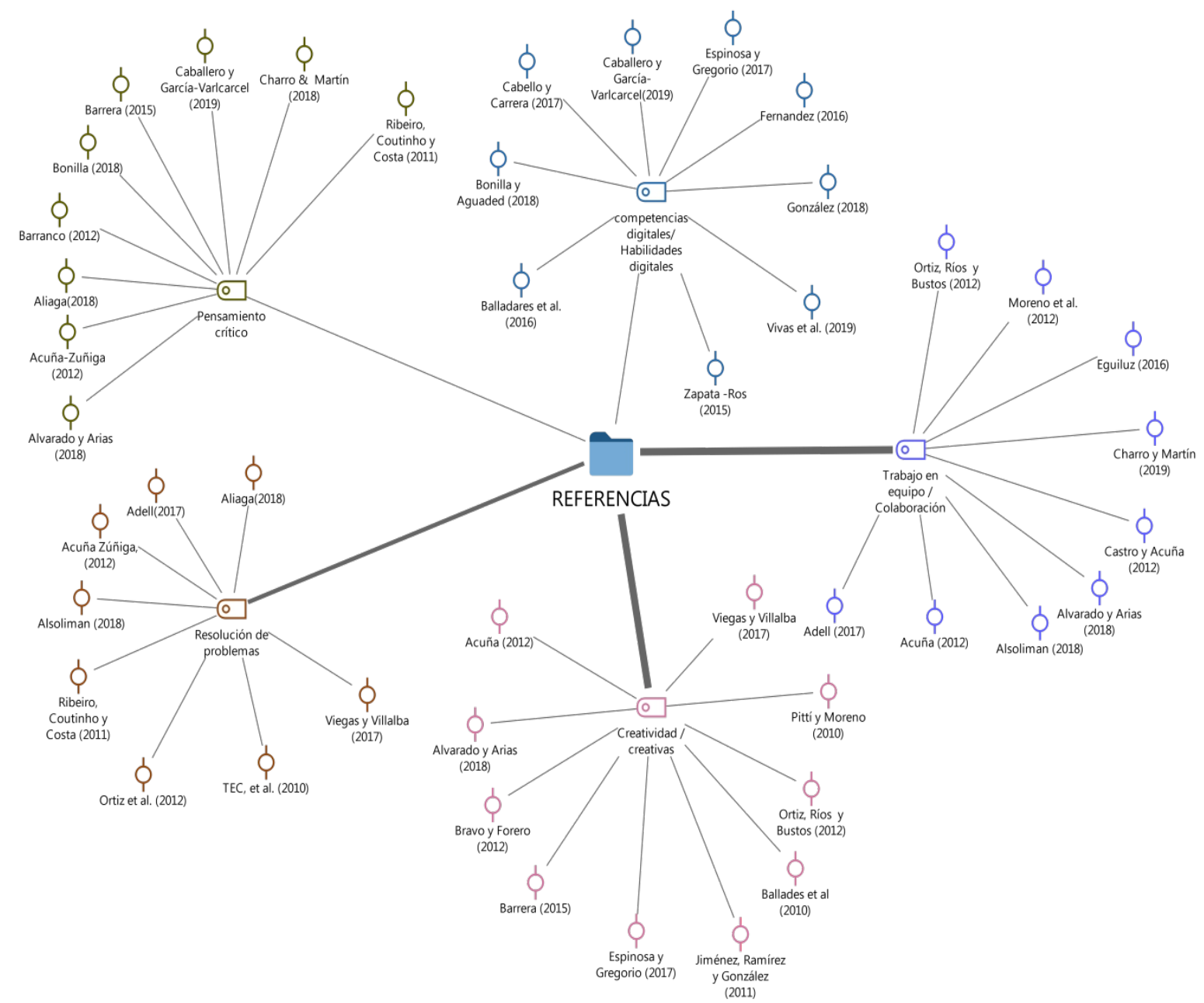

Figura 5. Habilidades relacionadas con la robótica educativa y referencias más significativas (Elaboración propia) 
Otra capacidad es el trabajo en equipo, al involucrar a los estudiantes en experiencias colaborativas. En este cometido el educando realiza una serie de tareas como el analizar y representar datos de manera lógica con la intención de dar solución a problemas (Adell 2017). Al mismo tiempo que se desarrolla un pensamiento crítico y espacial como es el caso del uso del robot Bee-Boot en el proyecto de Caballero y García-Varlcarcel (2019).

Es así que la robótica educativa actúa como un elemento que promueve el aprendizaje activo mediante un complejo de procesos cognitivos (percepción, presentación, imaginación, pensamiento, memoria y habla). Además de una gama de cualidades como: Motivación, sus iniciativas, el sentido de responsabilidad frente al trabajo, sociabilidad, tolerancia, lucha por el éxito, necesidad de autorrealización y habilidad de comunicación (Barrera 2015).

Todo esto dependerá del proyecto asignado, del reto que representa y que sea acorde al nivel de desarrollo de los participantes. Además de que la actividad del proyecto de robótica debe estar asociada con el contenido del proceso académico sobre una temática (Ospennikova et al. 2015).

En el estudio de Moreno et al. (2012) la robótica educativa es considerada como un elemento de mejora del aprendizaje, sin embargo, éste no es siempre el caso, ya que hay estudios que informaron situaciones en las que no hubo progreso en el aprendizaje y describen las barreras relativas al uso de la herramienta en el aula como son: la inversión en la tecnología, la constante actualización de los robots, la falta de conocimientos por parte de los docentes y la falta de estrategias de robótica educativa (Gjovik 2013, Kradolfer et al. 2014, Alsoliman 2018). Este último obstáculo se asocia con la capacitación docente en el uso de la tecnología y el desarrollo creativo para generar situaciones problema para que sean resueltos con una herramienta específica.

En la intervención de centros comunitarios realizada por Castro y Acuña (2012), la propuesta de robótica educativa presentó dificultades en el logro de habilidades interpersonales como el trabajo en equipo, la comunicación y socialización de los procesos de aprendizaje. Por lo que es necesario continuar con investigaciones para rescatar las buenas prácticas y generar metaanálisis de las mismas ya que en las últimas realizadas por Benitti (2012) encuentra resultados contradictorios.

\section{La robótica educativa y el aprendizaje STEAM}

Los artículos encontrados demuestran que el aprendizaje STEAM cobra más fuerza en niveles educativos tempranos. De acuerdo con González (2018) esto se debe a la necesidad de que los niños conozcan y comprendan conceptos del mundo altamente tecnificado y sistematizado que les rodea. Utilizar la robótica educativa como una vía para el logro de aprendizaje STEAM permite familiarizar al educando con el desarrollo tecnológico de una forma motivante y lúdica (Da Silva y González 2017). En diversas investigaciones se encontró dicho acrónimo y este puede ser abordado desde los conceptos de educación, aprendizaje, metodología y técnicas.

Desde un término amplio se habla de educación STEAM y centran su interés en defender la formación de habilidades y competencias en los estudiantes como es el caso de las investigaciones de (Ngamkajornwiwat et al. 2017, García y Caballero 2019, Charro y Martín 2019, Sánchez 2019). Mientras que existen las investigaciones que se centran en los procesos de aprendizaje de conocimientos STEAM (Ruiz 2017, Da Silva y González 2017, González 2018). Por último, los estudios que proponen metodologías y técnicas para la realización de talleres STEAM (Alvarado y Arias 2018, Rodríguez 2018). 
Las competencias asociadas al aprendizaje STEAM son: la autonomía y emprendimiento, colaboración, comunicación, conocimiento y uso de la tecnología, creatividad e innovación, diseño y fabricación de productos, pensamiento crítico y resolución de problemas (Sánchez Ludeña 2019). Estas capacidades pueden ser abordadas de forma transversal, desde distintas asignaturas del currículum. Una de las propuestas metodológicas que sugieren la mayoría de las investigaciones es el aprendizaje basado en proyectos y el basado en problemas tecnológicos. Otra forma es mediante tinkering (aprender haciendo) que a diferencia de las otras dos estrategias mencionadas no tiene un orden concreto de pasos para llegar a la solución de un problema, por lo que se presta para un aprendizaje de ensayo y error, esto ayuda a que la sensación de aprendizaje sea de forma lúdica.

Los documentos STEAM desde su abordaje pedagógico y metodológico se orientan al constructivismo y construccionismo como constante en diversas investigaciones, en donde se destaca la evolución del constructivismo al construccionismo de Papert y Harel (1991), a partir del principio piagetiano de que el individuo requiere de materiales para construir, centrándose en la persona y en el arte de aprender a aprender. Este principio se ha popularizado debido a que LEGO financió los proyectos de Papert con el uso de robots. Las metodologías asocian principios pedagógicos como involucrar y motivar a los estudiantes a la experimentación creativa e interacción social. En la tabla 2 se observa un resumen de las investigaciones sobre robótica, STEAM y su relación con aspectos didácticos, metodológicos y aspectos pedagógicos.

Tabla 2. Referencias STEAM a partir de la postura pedagógica - metodológica

\begin{tabular}{|c|c|}
\hline Referencia & Modelo pedagógico y metodología didáctica \\
\hline Alvarado y Arias (2018) & Estrategias lúdicas / constructivismo \\
\hline Cadorin, et al. (2017) & $\begin{array}{l}\text { Mobile Learning-Marco TPACK } \\
\text { Content knowledge) }\end{array}$ \\
\hline Charro y Martín (2018) & Interdisciplinar, ABP/ Constructivismo \\
\hline Chung (2014) & $\begin{array}{l}\text { Integración de la Robótica de forma natural para el desarrollo de } \\
\text { STEAM }\end{array}$ \\
\hline da Silva y González (2017) & $\begin{array}{l}\text { Aprendizaje Lúdico, / pensamiento computacional y ABP, } \\
\text { constructivismo y construccionismo. }\end{array}$ \\
\hline Fuentes y González (2017) & Gamificación \\
\hline Fuentes-Hurtado y González (2019) & Modelo TPACK y gamificación \\
\hline González (2018) & Aprendizaje vivencial/ constructivista, construccionista \\
\hline Hamner y Cross (2013) & Integración en el aula de Arts \& Bots \\
\hline Ngamkajornwiwat et al. (2017) & Herramientas convergentes, multidisciplinariedad \\
\hline REDEM (2018) & Aprendizaje lúdico \\
\hline Rodríguez (2018) & Aprendizaje interdisciplinar/pedagogía praxeológica \\
\hline Ruiz (2017) & $\begin{array}{l}\text { Metodología activa ABP y proyectos, praxeológica y aprendizaje } \\
\text { cooperativo }\end{array}$ \\
\hline Sánchez (2019) & Enfoque interdisciplinar, espacios Maker \\
\hline Villacís (2019) & ABP, multidisciplinar y Construccionismo \\
\hline Yakman y Lee (2012) & Constructivista, prácticas multidisciplinarias, aprendizaje integrador \\
\hline Zamorano, García y Reyes (2018) & $\begin{array}{l}\text { Interdisciplinar, aprendizaje basado en proyectos y en problemas/ } \\
\text { Construccionismo }\end{array}$ \\
\hline
\end{tabular}


Para Rodríguez (2008) “el construccionismo reconoce que la función primaria del lenguaje es la construcción de mundos humanos contextualizados... Lo que ocurre entre los seres humanos adquiere significado a partir de la interacción social expresada a través del lenguaje" (pág. 82). Por tal motivo los conocimientos se construyen activamente a través de contextualizar el entorno y sus relaciones con los demás, así como la significatividad que le causa al estudiante al actuar con asertividad (Aparicio y Ostos 2018). Por tal motivo el papel del docente es ser mediador entre el estudiante y el proceso de aprendizaje. Entonces el acompañamiento del docente es fundamental ante diversos problemas que le plantee que ayuden a generar sus propios descubrimientos.

Da Silva y González (2017) argumentan que la robótica educativa representa una herramienta para aplicar de manera propicia la filosofía construccionista y constructivista mediante metodologías activas Kotsopoulos et al. (2017) puesto que otorga al niño oportunidades para trasladar experiencias a un contexto determinado. Así permite conectar conocimientos previos a nuevos contextos a partir de sus representaciones internas y dar sentido a lo que se encuentra en su entorno. Todo esto a partir de experiencias y actividades basadas en la indagación y manipulación de materiales, en este caso el uso de diferentes elementos que se pueden integrar a los robots. Además, el uso de estas herramientas tecnológicas permite el desarrollo de un aprendizaje social y activo al colaborar e incentivar sus intereses, pensamiento crítico, creativo y analógico.

Hay autores que se oponen al aprendizaje memorístico de las ciencias (Cadorin et al. 2017, Fuentes y González 2017). Por lo que retoma el modelo de Technological Pedagogical Content Knowledge (TPACK) desarrollado por (Mishra y Koehler 2006, Koehler et al. 2015). Este modelo de integración de la tecnología por parte del docente, se centra en tres principios básicos que el docente desarrolla para construir estrategias de enseñanza como son: Contenido (CK), Pedagógico (PK) y Tecnológico (TK).

Otra de las metodologías que permite el aprendizaje STEAM es la gamificación que es una forma alternativa para enseñar, en que el estudiante se ve motivado a aprender detonando emociones positivas y propicia el desarrollo de habilidades como la creatividad, la curiosidad y el deseo por aprender (REDEM 2017, Alvarado y Arias 2018, Fuentes-Hurtado y González 2019).

Por último, es importante señalar que Sánchez (2019) prevé que son tres las dificultades al implementar la educación STEAM en la escuela como son: resistencias y problemas en la organización del sistema educativo; dotación y acceso a los recursos necesarios para llevar a cabo los proyectos y la formación del profesorado. En esta última limitante se destaca que el perfil del profesorado debe de ser muy específico además del conocimiento de tecnologías avanzadas y la capacidad para utilizar la transversalidad de las asignaturas.

\section{Desarrollo del pensamiento computacional en niños y adolescentes}

El pensamiento computacional está asociado a la robótica educativa porque permite aprender a resolver problemas mediante un enfoque algorítmico en diferentes niveles de abstracción (Ioannou y Makridou 2018). Estos autores descubrieron que además de este tipo de pensamiento, la robótica es una adecuada estrategia para promover habilidades cognitivas y sociales.

En este apartado se analizaron 18 documentos, de los cuales se destacan ocho investigaciones de tipo cualitativas, cuatitativas y casos de éxito, además de 10 trabajos documentales. Entre la tecnología más utilizada se encuentra el lenguaje visual Scratch y la robótica. La clasificación de estos documentos se puede observar en la tabla 3. 
Tabla 3. Estudios respecto al pensamiento computacional

\begin{tabular}{|c|c|c|c|}
\hline Referencia & Nivel & Tipo de estudio & Tecnología \\
\hline $\begin{array}{l}\text { Adell, Llopis Esteve, y } \\
\text { Valdeolivas (2019) }\end{array}$ & Educación básica & Investigación documental & No se específica \\
\hline $\begin{array}{l}\text { Balladares, Avilés y } \\
\text { Pérez (2016) }\end{array}$ & $\begin{array}{l}\text { Educación básica y } \\
\text { bachillerato }\end{array}$ & Investigación documental & No se específica \\
\hline $\begin{array}{l}\text { Barrera y Montaño } \\
(2015)\end{array}$ & Educación básica & Experiencia descriptiva & Scratch \\
\hline $\begin{array}{l}\text { Bonilla-del-Río y } \\
\text { Aguaded (2018) }\end{array}$ & Educación básica & Enfoque mixto & $\begin{array}{l}\text { Kit de robótica, LEGO } \\
\text { WeDo, Scratch } 2.0 \text { y el } \\
\text { recurso App Inventor }\end{array}$ \\
\hline Cadillo (2015) & Educación básica & $\begin{array}{l}\text { Cuantitativo (pre-experimental, } \\
\text { pre y post test) }\end{array}$ & No se específica \\
\hline Eguiluz (2016) & Educación infantil & Investigación documental & No se específica \\
\hline $\begin{array}{l}\text { Espino y González } \\
(2015)\end{array}$ & $\begin{array}{l}\text { Docentes y niños de } \\
\text { educación básica }\end{array}$ & $\begin{array}{l}\text { Cuantitativo estudio } \\
\text { comparativo }\end{array}$ & No se específica \\
\hline $\begin{array}{l}\text { García-Valcárcel y } \\
\text { Caballero (2019) }\end{array}$ & Educación infantil & Cuantitativo cuasi-experimental & BeeBot \\
\hline $\begin{array}{l}\text { Ioannou y Makridou } \\
\text { (2018) }\end{array}$ & Educación básica & Investigación documental & Ninguna \\
\hline $\begin{array}{l}\text { Kotsopoulos, y otros } \\
(2017)\end{array}$ & Educación básica & Investigación documental & Arduino y Scratch \\
\hline $\begin{array}{l}\text { Marmolejo y Campos } \\
(2012)\end{array}$ & Educación básica & Experiencia descriptiva & No se específica \\
\hline Monjelat (2019) & $\begin{array}{l}\text { Docentes, } \\
\text { educación básica }\end{array}$ & Cualitativo (estudios de casos) & Scratch \\
\hline Rincón y Ávila (2016) & No se especifica & Investigación documental & No se específica \\
\hline Rincón y Ávila (2016) & No se especifica & Investigación documental & No se específica \\
\hline $\begin{array}{l}\text { Santos y Osório } \\
(2019)\end{array}$ & $\begin{array}{l}\text { Nivel básico, niños, } \\
\text { docentes y padres }\end{array}$ & Cualitativa & Scratch y robótica \\
\hline Suárez et al. (2018) & Nivel primario & Investigación documental & LEGO \\
\hline $\begin{array}{l}\text { Valverde, Fernández y } \\
\text { Garrido (2015) }\end{array}$ & $\begin{array}{l}\text { Educación } \\
\text { secundaria }\end{array}$ & Investigación documental & Diseño curricular \\
\hline Zapata-Ros (2015) & Educación básica & Investigación documental & No se específica \\
\hline
\end{tabular}

Es importante rescatar la definición que Wing (2006) aporta, el "Pensamiento computacional incluye una variedad de herramientas mentales que reflejan amplitud del campo de la informática" (pág. 33). Mientras que Balladares et al. (2016) destacan que es un concepto complejo y responde a la necesidad de la sociedad actual para generar habilidades como resolver problemas de diferente tipo con ayuda de herramientas digitales e informáticas.

En este sentido se asocian el pensamiento computacional y las competencias digitales que los niños y jóvenes deben desarrollar en la sociedad actual (Zapata-Ros 2015, Valverde et al. 2015). 
Este tipo de pensamiento promueve la resolución de problemas en diferentes niveles de abstracción, al descartar la memorización y a partir de un conjunto de elementos: programas, videojuegos, robots, que juegan un papel al ser mediadores concretos para que los estudiantes puedan materializar su pensamiento, o modificarlo y enriquecerlo. Por tal motivo en el pensamiento computacional lo importante son las ideas que el estudiante genera para ser plasmadas en un artefacto.

Por el contrario, Adell et al. (2019) consideran que este concepto es incipiente y polémico, por lo que no hay una definición ampliamente aceptada y genera una crítica a las políticas educativas de integrar el pensamiento computacional en el currículum. Así mismo, se opone a que sea considerado como parte de las competencias digitales. Concluye que es necesario estudiar su aplicación como una habilidad más que requieren los estudiantes de nivel básico. Mientras que destaca que es un concepto complejo que responde a la necesidad de la sociedad actual para generar habilidades para resolver problemas de diferente tipo con ayuda de las herramientas digitales e informáticas.

Kotsopoulos et al. (2017) presentan un marco referencial pedagógico del pensamiento computacional pedagógico basado en el construccionismo y la teoría sociocultural de Vigotsky, de zona de desarrollo próximo para promover habilidades relacionadas con el pensamiento computacional.

Balladares et al. (2016) plantean una relación directa entre el pensamiento complejo y el computacional. Mientras que Barrera y Montaño (2015) asocian las habilidades de recopilación y representación de datos, modularización de problemas, abstracción y crear algoritmos y procedimientos.

Para finalizar mencionar el trabajo realizado por el Ministerio de Educación, Cultura y Deporte de España (MECD, 2018) que, en su informe sobre la enseñanza de la programación, robótica y pensamiento computacional en el aula, describen un conjunto de programas que han sido de éxito desde la educación infantil hasta el bachillerato. Más sin embargo se alude a la importancia de la capacitación docente en los logros de estas iniciativas.

\section{Conclusiones}

Las diversas investigaciones analizadas demuestran que actualmente se cuenta con experiencia documental referente a la robótica educativa. En este sentido se nota un avance por la cantidad de estudios que demuestran la efectividad del uso de dicha herramienta como mediadora del aprendizaje.

Se demuestra la evolución histórica de la robótica educativa, que en sus inicios se centró en aprendizajes de ciencias y matemáticas, pero conforme evoluciona se ha demostrado el beneficio que tienen para el aprendizaje STEAM. Los recientes estudios tienden a la interdisciplinariedad que beneficia no sólo a las ciencias mencionadas, sino al desarrollo de competencias como la autonomía y emprendimiento; la colaboración y comunicación; el uso de la tecnología; la creatividad e innovación, diseño y fabricación de productos; pensamiento crítico y resolución de problemas.

El papel del docente es fundamental para la implementación de cualquier tecnología, se sugiere formar a un profesor competente en pedagogía y con capacidades específicas según la tecnología a utilizar. Además, debe tener un pensamiento flexible-creativo e interdisciplinar para plantear el desarrollo de estrategias y configurar ambientes de aprendizaje favorables. Por tal motivo, las teorías pedagógicas hacen referencia a la evolución del constructivismo al construccionismo a partir del aprendizaje contextual y social. 
Se demuestra la necesidad de realizar un cambio en la enseñanza de ciencias, matemáticas y tecnología basado en principios de metodologías activas, al aplicar la gamificación y el trabajo colaborativo. Es importante mencionar que faltan estudios empíricos que muestren cómo se diseñaron las estrategias didácticas y los instrumentos para evaluar el impacto de la robótica educativa. Esta información se considera importante ya que permite orientar a docentes que están interesados en innovar sus prácticas. La tendencia es aplicar la robótica como un eje interdisciplinario que compagine los contenidos curriculares con los tecnológicos, que permita el desarrollo de habilidades para la vida de los alumnos.

Se encontraron muy pocos estudios con resultados negativos, sin embargo, son de gran utilidad para fortalecer futuras experiencias y preverlos al momento de incluir este tipo de actividades en el currículum. Esto es importante para avanzar en nuevos desarrollos tecnológicos y estrategias metodológicas para su enseñanza.

Son insuficientes las investigaciones en México que describen el fenómeno del pensamiento computacional y el aprendizaje STEAM. Se requiere realizar más estudios sistemáticos y evaluar los impactos que están teniendo los diversos programas e iniciativas gubernamentales de este tipo.

Es importante contar con estudios empíricos que demuestren las bondades del uso de estas herramientas tecnológicas, para que sean integradas de forma organizada en las políticas educativas de los países que apuesten por este tipo de preparación e integración en los currículums escolares.

\section{Referencias}

Acuña, A. L. S. (2012) Diseño y administración de proyectos de robótica educativa: lecciones aprendidas. Teoría de la Educación. Educación y Cultura en la Sociedad de la Información 13(3), 6-27

Adell, A. S., Llopis, M.Á.N., Esteve, F.M.M., Valdeolivas, M.G.N. (2019) El debate sobre el pensamiento computacional en educación. RIED.Revista Iberoamericana de Educación a Distancia 22(1), 171-186. Doi: http://dx.doi.org/10.5944/ried.22.1.22303

Aliaga, I.M.C., Carhuaricra, J.C., Asencio, L.V.T., Piñas, L.C.R. (2018) Programa de robótica educativa para mejorar el aprendizaje significativo en estudiantes del cuarto grado del área de Ciencia y Ambiente de la institución educativa. Revista EDUCA UMCH 11, pág-147. Doi: https://doi.org/10.35756/educaumch.v11i0.70

Alsoliman, B.S.H. (2018) The Utilization of Educational Robotics in Saudi Schools: Potentials and Barriers from the Perspective of Saudi Teachers. International Education Studies 11(10), 105-111.

Alvarado, D.S., Arias E.M. (2018) Experiencia STEAM. Proyecto Programación: La Nueva Alfabetización. Revista Atlante. Obtenido de https://www.researchgate.net/profile/Esteban_AriasMendez/publication/331288021_Experiencia_STEAM_-_Proyecto_Programacion_La_Nueva_Alfabetizacion/links/5c707dea458515831f67bc 31/Experiencia-STEAM--Proyecto-Programacion-La-Nueva-Alfabetizacion.pdf

Aparicio, O.Y.G., Ostos O.L.U. (2018) El constructivismo y el construccionismo. Revista interamericana de investigación, educación y pedagogía 2(11), 115-120. Doi: https://doi.org/10.15332/s1657-107X.2018.0002.05 
Balladares, B.J.A., Avilés M.R.S., Pérez, N.H.O. (2016) Del pensamiento complejo al pensamiento computacional: retos para la educación contemporánea. Sopbia, Colección de Filosofía de la Educación (21), 159.

Barranco, A.A.C. (2012) La robótica educativa, un nuevo reto para la educación panameña. Teoría de la Educación. Educación y Cultura en la Sociedad de la Información 13(2), 9-17.

Barrera, N.L. (2015) Uso de la robótica educativa como estrategia didáctica en el aula. Revista Praxis \& Saber 11(6), 215-234.

Barrera, R.C., Montaño, R.E. (2015) Desarrollo del Pensamiento Computacional con Scratch. Nuevas Ideas en Informática Educativa (pp. 616-620). Obtenido de http://www.tise.cl/volumen11/TISE2015/616-620.pdf

Benitti, F. B. V. (2012) Exploring the educational potential of robotics in schools: A systematic review. Computers \& Education 58(3), 978-988.

Bonilla-del-Río, M., Aguaded, I. (2018) La escuela en la era digital: smartphones, apps y programación en educación primaria y su repercusión en la competencia mediática del alumnado. Píxel-Bit. Revista de Medios y Educación (53), 151-163. Doi: http://dx.doi.org/10.12795/pixelbit.2018.i53.10

Bravo, F. Á.S., Forero, A.G. (2012) La robótica como un recurso para facilitar el aprendizaje y desarrollo de competencias generales. Revista Teoría de la Educación. Educación y Cultura en la Sociedad de la Información 13(2), 120-136.

Caballero, Y. A.G., García-Valcárcel, M.R.A. (2019) Fortaleciendo habilidades de pensamiento computacional en Educación Infantil: Experiencia de aprendizaje mediante interfaces tangible y gráfica. RELATEC: Revista Latinoamericana de Tecnología Educativa 18(2), 133150. Doi: http://dx.medra.org/10.17398/1695-288X.18.2.133

Cadillo León, J. R. (2015) Scratch y WeDo como herramientas para desarrollar el pensamiento computacional en niños de 11 y 12 años. Obtenido de https://reposital.cuaed.unam.mx:8443/xmlui/handle/20.500.12579/4186

Cadorin P. N., Sommer, S. M. B., da Silva, M. A.C., Schardosim J. P. S., da Silva, J. B. (2017) Technology Integration Actions in Mathematics teaching in Brazilian Basic Education: Stimulating STEM disciplines. Revista de Educación a Distancia 7(52), 11-22. Doi: http://dx.doi.org/10.6018/red/52/7

Candanedo, A.A. (2012) La robótica educativa, un nuevo reto para la educación panameña. Teoría de la Educación. Educación y Cultura en la Sociedad de la Información 13(2), 9-17.

Carme, J., Antoli, O.J. (2015) Spatial ability learning through educational robotics. Int J Technol Des Educ, 185-203.

Castro, M.D.R., Acuña, A.L.Z. (2012) Propuesta comunitaria con robótica educativa: valoración y resultados de aprendizaje. Teoría de la Educación. Educación y Cultura en la Sociedad de la Información 2(13), 91-119.

Charro, E., Martín, L. (2019) El papel de la robótica educativa en la adquisición de la competencia STEM (Science-Technology-Engineering-Mathematics). Revista Atlante: Cuadernos de Educación $y$ Desarrollo. Obtenido de https://www.eumed.net/rev/atlante/2018/02/robotica-educativa-stem.html

Chavarría, -problema. M., Saldaño, A. (2010) La robótica educativa como una innovativa interfaz educativa entre el alumno y una situación Didáctica y Educación 1(2), 1-12. 
Chung, C. C. (2014) Integrated STEAM Education through Global. 4th IEEE Integrated STEM Education Conference (pp. 978-984). Princeton: IEEE.

Da Silva, M.G.F., González, C.S.G. (2017) Pequebot: Propuesta de un sistema ludificado de robótica educativa para la educación infantil. Libro de Actas del V Congreso Internacional de Videojuegos $y$ Educación. Obtenido de https://riull.ull.es/xmlui/bitstream/handle/915/6677/CIVE17_paper_14.pdf? sequence $=1$ \&is Allowed $=\mathrm{y}$

Eguchi, A. (2014) Robotics as a learning tool for educational transformation. In Proceeding of 4th international workshop teaching robotics, teaching with robotics \& 5 th international conference robotics in education Padova (Italy).

Eguiluz, N.L. (2016) El pensamiento computacional en el aula. Revista Arista Digital (52), 8189.

Escalante, M., Montañez, T., González, C., y García, M. (2010) Matemáticas basadas en Proyectos, Software de Animación, Robots, Lenguajes de Programación y Cámara Digital. In Memorias del Congreso Iberoamericano de Informática Educativa 2010 (pp. 727-735).

Espino, E. E., González, C. S.G. (2015) Estudio sobre diferencias de género en las competencias y las estrategias educativas para el desarrollo del pensamiento computacional. RED. Revista de Educación a Distancia (46), 1 -20.

Espinosa, C.M., Gregorio M.O. (2017) La Robótica en Educación Infantil. Publicaciones Didácticas 90(1), 282-288.

Fernández, R.R. (2016) Evaluación de la autopercepción del nivel de competencia profesional docente especifica en robótica educativa (Master's thesis): Universitat de Lleida. Obtenido de https://repositori.udl.cat/bitstream/handle/10459.1/57985/rfernandezr.pdf? sequence $=1$ \&is Allowed $=\mathrm{y}$

Filipp, S., Ten, N., Shirokolobov, I., Fradkov, A. (2017) Teaching Robotics in Secondary School. ScienceDirect 50(1), 12155-12160.

Fuentes, M.H., González J.M. (2017) Necesidades formativas del profesorado de Secundaria para la implementación de experiencias gamificadas en STEM. RED. Revista de Educación a Distancia (54), 1-25. Doi: http://dx.doi.org/10.6018/red/54/8

Fuentes, M.H., González, J.M. (2019) Evaluación inicial del diseño de unidades didácticas STEM gamificadas con TIC. Edutec. Revista Electrónica De Tecnología Educativa (70), 1 17. Doi: https://doi.org/10.21556/edutec.2019.70.1469

Galeano, M.E.M., Vélez, O.L.R. (2002) Estado del arte sobre fuentes documentales en Investigación cualitativa. Medellín: Universidad de Antioquia. Centro de Investigaciones Sociales y Humanas.

García, J. M. (2015) Robótica Educativa. La programación como parte de un proceso educativo. Revista de Educación a Distancia. RED 8 (46), 1-11. Doi: 10.6018/red/46/8

García-Valcárcel, A.M.R. Caballero Y.A.G. (2019) Robótica para desarrollar el pensamiento computacional en Educación Infantil. Comunicar: Revista cientifica iberoamericana de comunicación y educación (59), 63-72. Doi: https://doi.org/10.3916/C59-2019-06

Ghitis, T.J., Alba, J.V. (2014) Los robots llegan a las aulas. Revista Infancias Imágenes 13(1), 143 147. 
Girvan, C., Lammer, L., Lepuschitz, W., Kynigos, C., Giuliano, A. (2017) ER4STEM robótica educativa para ciencia, tecnología, ingeniería y matemáticas. En Robótica en educación (pp. 95-101). Springer, Cham.

Gjovik, K.P. (2013) Determining factors that serve as barriers to integrative STEM methodology implementation in K-12 schools (Doctoral dissertation, North Dakota State University).

Obtenido

de https://library.ndsu.edu/ir/bitstream/handle/10365/27180/Determining\%20Factors $\% 20$ that $\% 20$ Serve $\% 20$ as $\% 20$ Barriers $\% 20$ to $\% 20$ Integrative $\% 20$ Stem $\% 20$ Methodology $\% 20$ Implementation $\% 20 \mathrm{in} \% 20 \mathrm{~K}-12 \% 20$ Schools.pdf?sequence $=1 \&$ is Allowed $=\mathrm{y}$

Gómez, G.H.V. (2015) El Estado del Arte: una metodología de investigación. Revista Colombiana de Ciencias Sociales 6(2), 423-442.

González, C.S.G. (2018) La enseñanza-aprendizaje del Pensamiento Computacional en edades tempranas: una revisión del estado del arte. En Pensamiento computacional. Zapata-Ros M. y Villalba Condor K. O. (Coordinadores). Editorial Universidad Católica de Santa María de Arequipa, Perú.

Hamner, E. y Cross, J. (2013) Arts \& Bots: Techniques for Distributing a STEAM Robotics Program Through K-12 Classrooms. 3rd IEEE Integrated STEM Education Conference (pp. 12). Princeton, NJ: IEEE.

Herrera,Y.C. H., Rincon, D.L. (2013) Estado del arte de la robótica educativa en el ámbito mundial.

Obtenido

de https://repository.uniminuto.edu/bitstream/handle/10656/2396/TI_HerreraHuertas Yudi_2012.pdf? sequence $=1$ \&isAllowed $=\mathrm{y}$

Ioannou, A., Makridou, E. (2018) Exploring the potentials of educational robotics in the development of computational thinking: A summary of current research and practical proposal for future work. Educ Inf Technol (23), 531-2544. Doi: https://doi.org/10.1007/s10639-018-9729-z

Jiménez, J.A.B., Ramírez, J.P., González, J.E. (2011) Sistema modular de robótica colaborativa aplicado en educación. Revista Facultad de Ingeniería Universidad de Antioquia (58), 163-172.

Khanlari, A. (2016) Teachers' perceptions of the benefits and the challenges of integrating educational robots into primary/elementary curricula. European Journal of Engineering Education 41(3), 320-330. Doi: https://doi.org/10.1080/03043797.2015.1056106

Koehler, M.J., Mishra, P., Cain, W. (2015) ¿Qué son los saberes tecnológicos y pedagógicos del contenido (TPACK)?. Virtualidad, educación y ciencia 6(10), 9-23.

Kotsopoulos, D., Floyd, L., Khan, S., Kizito, I.N., Somanath, S., Weber, J., Yiu, C. (2017) A Pedagogical Framework for Computational Thinking. Digit Exp Math Educ 3 (2), 154171. Doi: https://link.springer.com/content/pdf/10.1007/s40751-017-0031-2.pdf

Kradolfer, S., Dubois, S., Riedo, F., Mondada, F., Fassa, F. (2014) A sociological contribution to understanding the use of robots in schools: the thymio robot. In International Conference on Social Robotics. (pp.217-228). Springer, Cham.

Londoño, O.L.R, Maldonado L.F.G., Calderón L.C.V. (2016) Guía para construir estados de arte. Internarional Corporation of Networks of Knowledge (ICONK). Bogotá. Obtenido de http://iconk.org/docs/guiaea.pdf

López, P.A.R. y Andrade, H.S. (2013) Aprendizaje con robótica, algunas experiencias. Educación 37(1), 43-63. 
Mancilla, V.H.G., Aguilar, R.E.D., Aguilera, J.G.G., Subías, K.A., Ramírez, A.R. (2017) Robótica educativa para enseñanza de las ciencias. Revista Electrónica sobre Tecnología Educación y Sociedad, 4(7), 1-13.

Marmolejo, J.E.V. y Campos Salgado, V. (2012) Pensamiento lógico matemático con scratch en nivel básico. Vinculos 9(1), 87-95.

Márquez, J.E.D., Ruíz, J.H.F. (2014) Robótica educativa aplicada a la enseñanza básica secundaria. Revista cientifica de opinión y divulgación DIM (10) 30, 1-11.

Mataric M.J., Koenig, N., Feil-Seifer, D. (2007) Materials for Enabling Hands-On Robotics and STEM Education. Socially Assistive Robotics Group. Simposio de Primavera de AAAI sobre Robots y Lugares de Robots.

MECD Ministerio de Educación, Cultura y Deporte (2018) Programación, robótica y pensamiento computacional en el aula. Madrid: Instituto Nacional de Tecnologías Educativas y de Formación del Profesorado.

Mesa, L.A.M., Barrera, N.L. (2013) La robótica educativa como instrumento didáctico alternativo en educación básica. Revista colombiana de tecnologías de avanzada (RCTA) 2(22). Doi: https://doi.org/10.24054/16927257.v22.n22.2013.411

Mishra, P. y Koehler, M.J. (2006) Technological pedagogical content knowledge: A framework for integrating technology in teacher knowledge. Teachers College Record 108 (6), 10171054.

Monjelat, N. (2019) Programación de tecnologías para la inclusión social con Scratch: Prácticas sobre el pensamiento computacional en la formación docente. Revista Electrónica Educare 23(3), 1-25. Doi: https://doi.org/10.15359/ree.23-3.9

Moreno, I., Muñoz, L., Serracín, J.R., Quintero, J., Pittí K.P., Quiel, J. (2012) La robótica educativa, una herramienta para la enseñanza-aprendizaje de las ciencias y las tecnologías. Teoría de la Educación. Educación y Cultura en la Sociedad de la Información 13(2), 74-90.

Mubin, O., Stevens, C.J., Shahid, S., Al-Mahmud, A., Dong, J. (2013) A review of the applicability of robots in education. Technology for Education and Learning 1, 1-7.

Ngamkajornwiwat, P., Pataranutaporn, P., Surareungchai, W., Ngamarunchot, B., Suwinyattichaiporn, T. (2017) Understanding the role of arts and humanities in social robotics design: an experiment for STEAM enrichment program in Thailand. 6th International Conference on Teaching, Assessment and Learning for Engineering (TALE) (pp. 457-517). Hong Kong, China: IEEE.

Ocaña, G.R. (2012) Robótica como asignatura en enseñanza secundaria. Resultados de una experiencia educativa. Espiral. Cuadernos del profesorado 5(10), 56-64.

Ortiz, J.G.M., Ríos, A. R., Bustos, R.A.G. (2012) Laboratorio móvil tecno educativo: cursos de robótica de bajo costo para la alfabetización científica y tecnológica. Teoría de la Educación. Educación y Cultura en la Sociedad de la Información 13(3), 145-161.

Ospennikova, E., Ershov, M., Iljin, I. (2015) Educational robotics as an inovative educational technology. Procedia-Social and Behavioral Sciences 214, 18-26.

Papert, S., Harel, I. (1991) Situating constructionism. Constructionism 36(2), 1-11.

Pittí, K.P, Curto B.D., Moreno, V.R. (2010) Experiencias construccionistas con robótica educativa en el centro internacional de tecnologías avanzadas. Teoría de la Educación. Educación y Cultura en la Sociedad de la Información 11(1), 310-329. 
Pittí K.P., Curto, D.B., Moreno, V.R., Rodríguez, M. J.C., Rodríguez, J. F.A. (2014) Using Robotics as a Learning Tool in Latin America and Spain. IEEE revista iberoamericana de tecnologías del aprendiraje 9(4).

Plaza, P., Carro, G., Blazquez, M., Sancristobal, E., Castro, M., García-Loro, F., Muñoz, J. (2018) Crumble as an educational tool to introduce robotics. In 2018 XIII Technologies Applied to Electronics Teaching Conference (TAEE) (pp. 1-7). IEEE.

Rädiker S., Kuckartz U. (2019) Análisis de datos Cualitativos con MAXQDA Texto, Audio, video. Berlin, Germany: MAXQDA PRESS.

REDEM. (2017) La robótica educativa ya ha conquistado las aulas. Obtenido de https://www.redem.org/la-robotica-educativa-ya-ha-conquistado-las-aulas/

Ribeiro, C., Coutinho, C., Costa, M.F. (2011) La Robótica Educativa como Herramienta Pedagógica en la Resolución de Problemas de Matemáticas en la Enseñanza básica. CISTI.

Rincón, A.I.R., Ávila, W.D. (2016) Una aproximación desde la lógica de la educación al pensamiento computacional. Sophia, Colección de Filosofía de la Educación (21), 61- 176.

Rodríguez, C. (2018) Propuesta pedagógica fundamentada en la metodología STEAM para fortalecer el aprendizaje rizomático de los estudiantes de básica primaria. Proyecto OCTOPUS. Obtenido de https://repository.uniminuto.edu/handle/10656/6421

Rodríguez, H.V. (2008) Del constructivismo al construccionismo: implicaciones educativas. Revista Educación y Desarrollo Social (1), 71-89.

Romero, C., Nieto, J., Ochoa, C. (2014) Revisión del estado del arte de las plataformas robóticas orientadas a la educación. Journal of Engineering and Technology 3(2), 23-35.

Ruíz, F.V. (2017) Diseño de proyectos STEAM a partir del currículum actual de Educación Primaria utilizando Aprendizaje Basado en Problemas. Aprendizaje Cooperativo, Flipped Classroom y Robótica Educativa, Valencia: Universidad CEU Cardenal Herrera.

Sánchez, E.L. (2019) La educación STEAM y la cultura «maken». Journal of Parents and Teachers (379), 45-51.

Santos, M.P., Osório, A. (2019) Aprender a programar en educación infantil: análisis con la escala de participación. Píxel-BIT Revista de Medios y Educación (55), 133-156.

Suárez, A.Z., García, D.C., Martínez, P.A. D., Martos J.T. (2018) Contribución de la robótica educativa en la adquisición de conocimientos de matemáticas en la Educación Primaria. Magister: Revista miscelánea de investigación 30(1), 43-54. https:/ / dialnet.unirioja.es/servlet/articulo? codigo $=6722243$

Tec, B., Uc, J., González, C., García, M., Escalante, M., Mantañez, T. (2010) Análisis comparativo de dos formas de enseñar matemáticas básicas: robots legos nxt y animación con scratch. In Memorias de la Conferencia Conjunta Iberoamericana sobre Tecnologias para el Aprendizaje (pp. 103-109).

Valverde, J.B., Fernández M.R.S. y Garrido, M.D.C.A. (2015) El pensamiento computacional y las nuevas ecologías del aprendizaje. Revista de Educación a Distancia (46).

Viegas, J.V.D., Villalba, K.O.C. (2017) Educación y Robótica Educativa. RED (54), 1-13. Doi: http://dx.doi.org/10.6018/red/54/11 
Villacís, J.A.S. (2019) Integración de la robótica mediante el uso de la plataforma Arduino para el aprendizaje de matemáticas en el aula (Doctoral dissertation)Instituto Politécnico de Leira.Leira, Portuagal.

Wing, J.M. (March 2006) Computational Thinking. It represents a universally applicable attitude and skill set everyone, not just computer scientists, would be eager to learn and use. Communications of the ACM. 49 (3). Obtenido de https://www.cs.cmu.edu/ 15110s13/Wing06-ct.pdf

Yakman, G. (2008) ST $@ @ M$ Education: an overview of creating a model of integrative education. Pupils Attitudes Towards Technology. 2008 Annual Proceedings. Netherlands.

Yakman G., Lee, H. (2012) Exploring the Exemplary STEAM Education in the U.S. as a Practical Educational Framework for Korea. Journal of The Korean Association For Science Education 6(32), 1072-1086.

Zamorano, G.C.E. (2018) Educación para el sujeto del siglo XXI: principales características del enfoque STEAM desde la mirada educacional. Revista Contextos.Estudios De Humanidades $Y$ Ciencias Sociales, http://revistas.umce.cl/index.php/contextos/article/view/1395

(41).

Zapata-Ros, M. (2015) Pensamiento computacional: Una nueva alfabetización digital. REDRevista de Educación a Distancia 46(4). Doi : 10.6018/red/46/4 http://www.um.es/ead/red/46/zapata.pdf

Zúñiga, A.L. (2012) Diseño y administración de proyectos de robótica educativa: lecciones aprendidas. Teoria de la Educación. Educación y Cultura en la Sociedad de la Información 13(3), 6-27. 